Reporte de casos

\title{
Mucocele de labio: reporte de caso
}

Wilbert-Chirinos-Llerena ${ }^{1}$

\section{Resumen}

Paciente de sexo femenino, de 6 años de edad, que acude en compañía de su madre al servicio de medicina por molestias en el labio inferior, la menor es transferida al servicio dental. Al examen clínico se observa; una formación tumoral en la cara interna del labio inferior de un diámetro aproximado entre $5 \mathrm{~mm}$ a $7 \mathrm{~mm}$, y a la palpación; una consistencia blanda, elástica; de más de un año de evolución según narra la madre. Dadas las características clínicas de la lesión, nuestro diagnóstico presuntivo fue: $\mathrm{Mu}$ cocele de labio. fué evaluado histopatológicamente confirmándose el diagnóstico presuntivo.

Palabras Clave: Mucocele, labio, niños.

Relato de caso

\section{Mucocele labial: relato de caso}

\section{Resumo}

Paciente de sexo feminino, de 6 anos de idade, que procura, em companhia de sua mãe, o serviço de medicina devido a lesão no lábio inferior, sendo encaminhada ao serviço odontológico. No exame clínico observa-se; uma formação tumoral na face interna do lábio inferior com diâmetro aproximado entre $5 \mathrm{~mm}$ a
$7 \mathrm{~mm}$, apresentando na palpação consistência macia, elástica. Segundo relato materno, com evolução a mais de um ano. Considerando as características clínicas da lesão, nossa hipótese diagnóstica foi: mucocele labial. A avaliação histopatológica confirmou a hipótese diagnóstica.

Palavras chave: mucocele, lábio, criança.

\section{Case report}

\section{Lip Mucocele: case report}

\section{Summary}

A female patient, 6 years old, who came with her mother to the medical service as she had some discomfort in the lower lip; the minor is transferred to the dental service. The clinical examination shows: a tumoral formation on the internal side of the lower lip, with a diameter of approxi- 
mately between $5 \mathrm{~mm}$ and $7 \mathrm{~mm}$, and on palpation, it has a soft, elastic consistence; with over a year evolving; our presumed diagnosis was: Lip Mucocele. It was evaluated histopathologically, which confirmed the presumed diagnosis.

Key words: Mucocele, lip, children.

\section{Introducción}

El mucocele conocido también como fenómeno de retención mucosa, es una cavidad llena de moco, de tumoración nodular producida por algún traumatismo a la mucosa y glándulas salivales menores. El odontólogo general debe conocer los medios de diagnóstico a emplearse cuando se observa una lesión en tejidos blandos refiriéndonos a un quiste de partes blandas a diferencia de los quistes óseos que requieren de un exámen radiográfico. Teóricamente un quiste es una cavidad rodeada por una membrana de origen mesodérmico bien definida con una capa intermedia y una interna de origen ectodérmico, generalmente formada por epitelio de origen malphigiano poliestratificado o ciliado, a excepción de los mucoceles y ranulas, en los que generalmente se observa una capa externa conjuntiva; sin ninguna abertura, que contiene en su interior un material liquido o semiliquido de etiología desconocida, opinándose que viene de los restos ectodérmicos (vaina de Hertwig), o de inclusiones en los puntos de unión primarios de los procesos formativos de las estructuras maxilofaciales. $(1,2)$

En los quistes que aparecen en las zonas típicas del suelo de la boca, lengua labios, cuello y región de las glándulas salivales se constituye una tumoración lenta, indolora y redondeada de las partes blandas. La consistencia de esta tumoración va desde blanda a elástica, pudiéndose sentir ocasionalmente una fluctuación.
Los quistes situados en el tejido submucoso de la boca se ven a través de la mucosa con una coloración azulada o rojo azulada (quistes mucosos), o bien amarillenta (quiste dermoide). En lo referente a su crecimiento, como ya lo mencionamos anteriormente es muy lento, debido a la presión osmótica del liquido o semiliquido de su contenido, liquido que puede aumentar por osmosis a través de la capa conjuntivo-epitelial o por liquefacción de las células desprendidas de la capa interna del quiste. $(3,4,5,6,16)$

\section{Etiología y Patogenia}

Los mucoceles, suelen aparecer sobre todo en niños y adultos jóvenes aunque pueden hacerlo en cualquier edad. Casi la mitad de los casos se presentan antes de los 20 años de edad, mas de la cuarta parte entre las edades de 11 y 12 años, afectando por igual a hombres como a mujeres. La causa de este fenómeno se relaciona con traumatismo mecánicos de los conductos de las glándulas salivales menores que corta o desgarra dicho conducto, dando lugar a un derrame o extravasación de moco en el estroma de tejido conectivo circundante, donde precipita una reacción inflamatoria secundaria que al principio contiene neutrófilos y después macrófagos. El tejido de granulación presente favorece la formación de una pared alrededor de la mucina derramada. El tejido de la glándula salival adyacente sufre una alteración inflamatoria inespecífica a causa de la retención de moco y posterior cicatrización en el interior y alrededor de la glándula. $(7,3,14,15)$

\section{Características clínicas}

La retención mucosa se considera quiste porque, a diferencia de la extravasación de musina rodeada por tejido de granulación, esta cubierto 
por epitelio. El termino "mucocele" se utiliza en forma genérica antes de establecer el diagnostico microscópico, para referirse tanto al quiste por retención mucosa como al fenómeno de extravasación. $(1,2,3,10,15)$

Los mucoceles son relativamente frecuentes, pero algo importante que hay que tomar en cuenta es que el mucocele por retención es menos frecuente que el de extravasación. Probablemente las lesiones mecánicas o inflamatorias de pequeñas glándulas mucosas o salivales son la causa primordial. La localización más frecuente es el labio inferior pero también se puede encontrar en el paladar, carrillo, lengua (afecta a las glándulas de Blandin-Nuhn), y en el piso de la boca. $(1,8,9,10)$ Raras veces se encuentran en otras regiones de la boca donde se localizan glándulas salivales, tal vez por la ausencia relativa de traumatismos en estas regiones. $(6,8)$

Aparecen como tumoraciones únicas o múltiples con aspecto característico como lo son grisáceas y hasta casi trasparentes, la lesión puede estar situada profundamente en el tejido, o ser muy superficial y dependiendo de su localización presentaran un aspecto variable.

La lesión superficial aparece como una vesícula circunscrita de varios milímetros ó centímetros de diámetro (el tamaño de la misma se modifica de forma periódica), con un tinte traslucido de color azulado. La lesión más profundas se manifiesta también como un abultamiento, pero debido al grosor del tejido que lo cubre, el color y el aspecto de la superficie son los de la mucosa normal. $(6,11,12,13,16)$

\section{Histopatología}

Microscópicamente se muestra un quiste fibroso, revestido por células epiteliales del conducto, la gran mayoría de las cuales son oncocitos, que forman una capa doble seudoestratifica de células cuboidales o columnares. Como casi todos los mucoceles son del tipo extravasación, los cuales están formados por una cavidad circunscrita en el tejido conectivo y la submucosa que produce una elevación apreciable de la mucosa con adelgazamiento del epitelio. El epitelio superficial esta distendido por él acumulo de mucina y la cavidad en realidad no esta revestida por epitelio y por lo tanto no es un quiste verdadero.

La luz del quiste contiene cantidades variables de secreción viscosa o espesa y las células que principalmente se encuentran son leucocitos y fagocitos mononucleares.

El mucocele por retención se caracteriza por una cubierta derivada del epitelio de los conductos y también puede observarse un calculo salival (sioalolito), constituido por capas acelulares concéntricas. $(8,9,10,11,14,15)$

\section{Diagnóstico diferencial}

- Carcinoma Adenoquistico: Se presenta entre la 5ta. y 6ta. década de vida, el paciente refiere dolor local y presenta linfoadenopatia, además de producir metástasis hacia los ganglios cervicales en un 30\% de los casos.

- Carcinoma Mucoepidermoide: Es de crecimiento rápido, produce dolor local, se presenta generalmente entre la 3era. y 4ta. década de vida, el paciente presenta linfoadenopatia y produce metástasis.

- Tumor Benigno Mixto: Es más frecuente en mujeres que en hombres (6:4), se presenta entre la 4ta. y 6ta. década de vida, el paciente refiere molestia en la zona y raras vez obtiene tamaño mayores de 1 a $2 \mathrm{~cm}$. de diámetro. 
- Ránula: Tumor quistico blando, de liquido que se forma debajo de la lengua por obstrucción y dilatación de una de las glándulas salivales de la región, asi mismo podríamos mencionar, Fenómeno de la extravasación de moco, Malformación vascular (Neurofibroma), Lipoma y Quiste de erupción o Quiste gingival cuando surgen en la mucosa alveolar.

\section{Tratamiento}

Es la excisión quirúrgica total de la glándula involucrada para minimizar el riesgo de recidiva, A veces hay recurrencia después de la excisión, ya que evacuado su contenido se vuelve a llenar de nuevo, tan pronto como cicatriza la lesión. Si el tratamiento se efectúa de una manera adecuada y se eliminan las glándulas involucradas no se produce recurrencia de la lesión; por lo cual se indica la excisión de cualquier elemento acinico que se encuentre a fin de evitar extravasación mucosa post-operatoria en el sitio de la cirugía. $(6,9,12,13,14,15,16)$

\section{Presentación de caso}

Reportamos el caso clínico de una niña de 6 años de edad que acude en compañía de su madre al

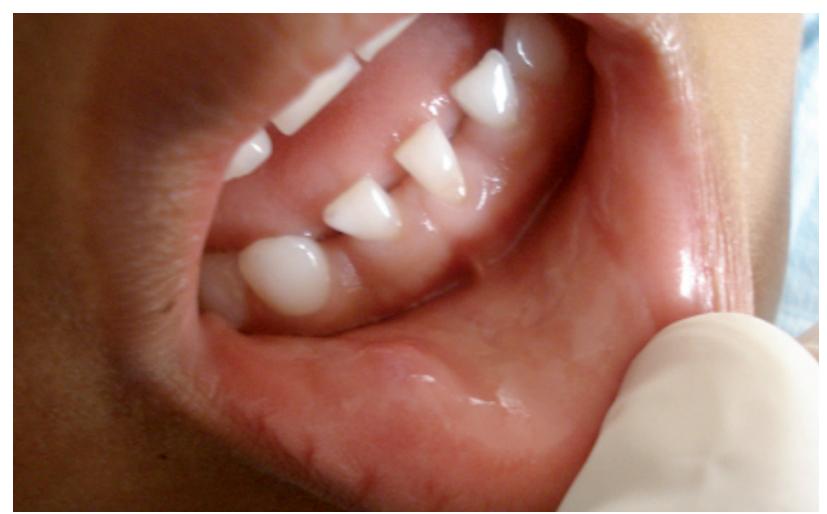

servicio de medicina del centro de salud de su zona, por molestias en el labio inferior, quien es referida al servicio dental. El motivo de la consulta es la presencia de un "bulto en el labio inferior que lo tiene más de un año y no desaparece" lo que molesta a la niña a la hora de comer sus alimentos, no hay dolor y no recuerda como apareció.

\section{Examen clínico bucal}

Se observa en la mucosa interna del labio inferior una tumoración elevada, de $5 \mathrm{~mm}$ a $7 \mathrm{~mm}$ de diámetro aproximadamente, de color de la mucosa bucal y aspecto brillante, a la palpación presenta consistencia blanda con un tiempo de evolución de más de un año (figura 1 y 2). Historia médica: buena salud general sin consideraciones a resaltar.

\section{Tratamiento}

El tratamiento recomendado es la excisión quirúrgica.

Exámenes previos al acto quirúrgico:

1. Pruebas hematológicas (tiempo de sangría, tiempo de coagulación): valores normales.

2. Interconsulta con Pediatría: Sin riesgo.

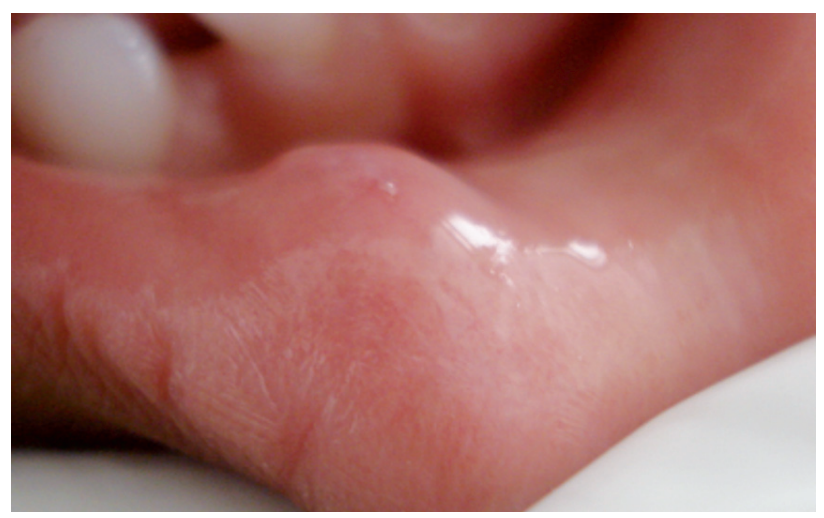

Fig. 1 y 2: Examen clínico de mucosa labial Se observa tumoración y consistencia fluctuante con diagnóstico presuntivo de mucocele labial. 


\section{Procedimiento quirúrgico}

El tratamiento que se realizó mediante biopsia excisional de la lesión debido a su tamaño pequeño se colocó anestesia infiltrativa alrededor de la lesión en 4 puntos cardinales, (arriba, abajo, derecha e izquierda) La cantidad de anestesia fue de $0.8 \mathrm{ml}$ para evitar edematizar la muestra y así poder tener los limites certeros de la lesión.

Con bisturí $\mathrm{n}^{\circ} 12$ se hizo una incisión recta sobre el tejido mucoso (figura 3) y en forma de cono hacia la profundidad del tejido (figura 4) para luego retirar toda la glándula comprometida (figura 5), para luego colocarla en un frasco con formol al $10 \%$ para su estudio histopatológico. Seguidamente procedimos a la sutura (hilo seda 4 ceros) colocando cuatro puntos (figura 6) y farmacoterapia correspondiente a Ibuprofeno tabletas de $200 \mathrm{mg}$ cada 8 horas y dicloxacilina tabletas de $250 \mathrm{mg}$ cada 12 horas por 5 días e indicaciones post-cirugía en la dieta no alimentos irritativos, e higiene bucal diaria complementada con enjuagatorios bucales.

Retiro de puntos a los 5 días, control y evaluación posteriores cada 6 meses (figura 7 y 8).

\section{Resultado histopatológico}

Hiperplasia pseudo-epiteliomatosa con neovascularizacion, inflamación crónica activa con presencia de neutrófilos e histiocitos, musina rodeado de Tejido granular moderada y negativo de malignidad.
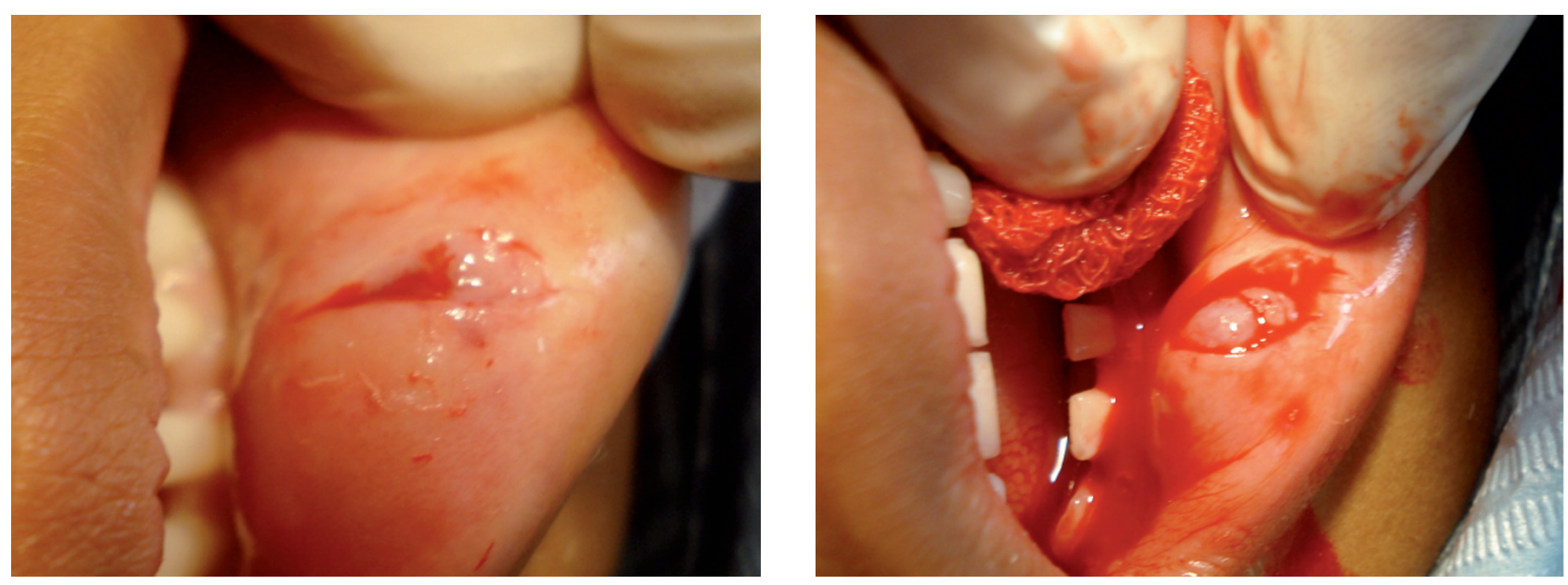

Fig. 3 y 4: Procedimiento Quirúrgico Incisión para exposición de la glándula a retirar.
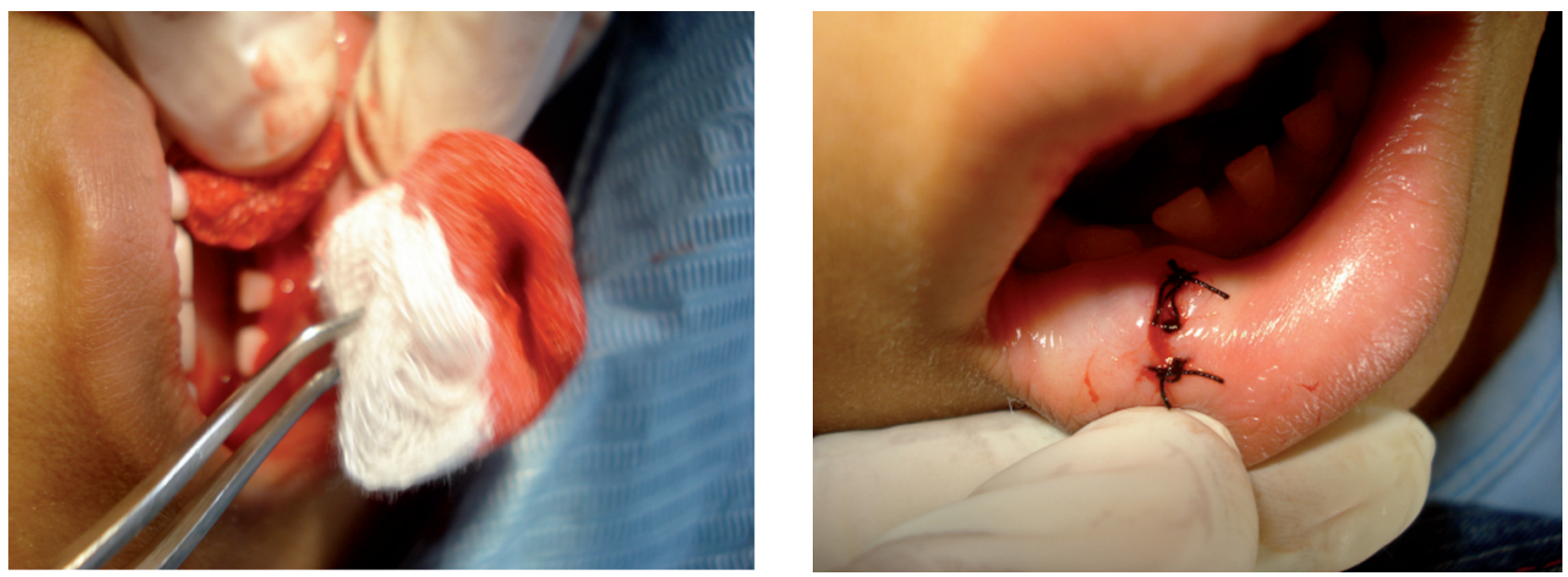

Fig. 5 y 6: Procedimiento Quirúrgico retiro de la glándula y sutura. 

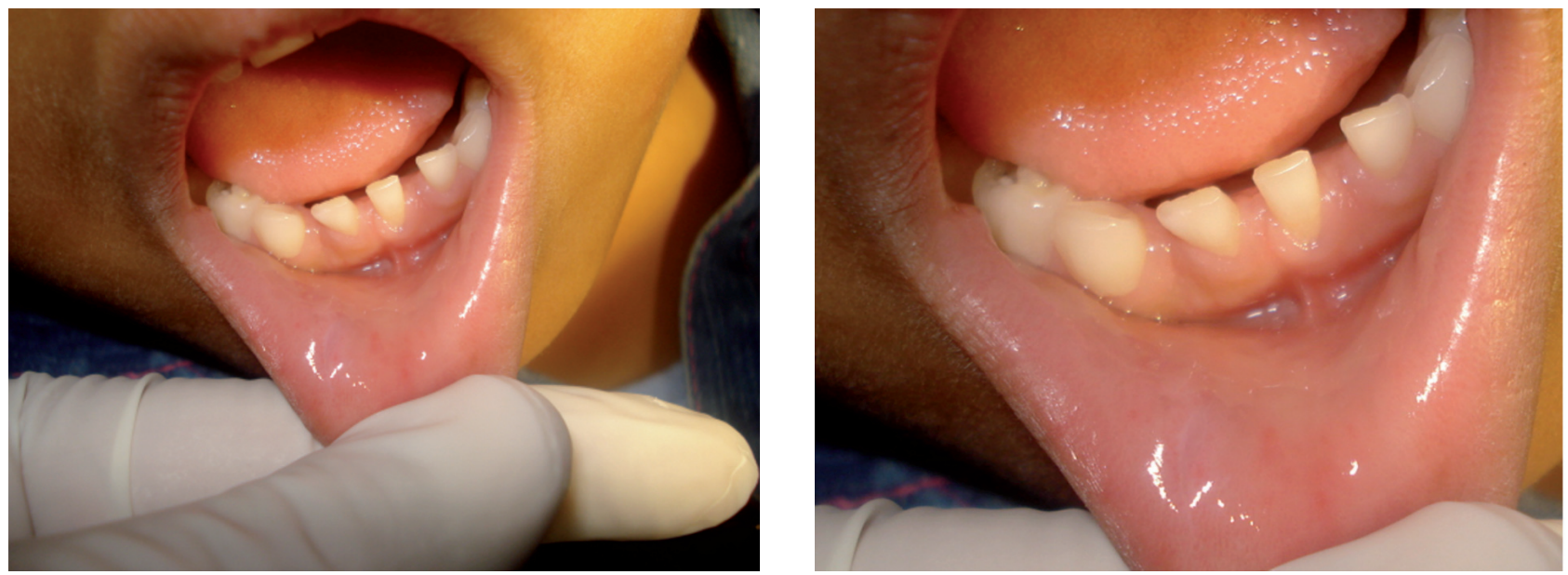

Fig. 7 y 8: Post-operatorio retiro de puntos y control a los 6 meses.

\section{Diagnóstico definitivo}

Diagnóstico definitivo MUCOCELE DE LABIO.

\section{Discusión}

El mucocele, es una patología benigna que se relaciona con el traumatismo, queilofagia, ó succión labial, está en los odontopediátras identificar el factor traumático y controlar él habito.

La ubicación por excelencia del mucocele es el labio inferior. En el momento de la excisión quirúrgica es necesaria la eliminación total de la lesión para evitar la recidiva y se debe eliminar el resto de las glándulas circundantes al mucocele.

\section{Conclusión}

La inspección visual es sólo un diagnóstico presuntivo o provisional, debe realizarse su correspondiente estudio histopatológico para obtener él diagnóstico definitivo.

\section{Referencias}

1. Anderson W.A.T. Pathology, 6th edition, st. Louis c.v. Mosby Company 1971

2. Arendorf tm .van wyk cw “ The association between perioral and mucoceles” Int J Oral Surg 1981 oct. 10:5 328-32.

3. de Lucas Tomás, Miguel. Atlas de medicina oral y maxilo-facial. Editorial Científico-Médica, 1985

4. Eversole, Roy: Patología bucal, edit. Ateneo. 1975.

5. Kruger: Cirugía buco-maxilofacial, Edit. Panamericana. 1986

6. Pinborg, JJ: Atlas de enfermedades de la mucosa oral, Edit. Salvat, 1981

7. Sapp J, Philip, Eversole, Lewis. Wysocki, George. Patología Oral y Maxilofacial Contemporánea. 1era. Edición. 1993. Harwurt, pp. 321-22

8. Giunta, J. L. Patología Bucal. 3era edición. 1.991. Interamericana Mc Graw - Hill. Argentina., pp122-24.

9. Lovas JG, Goodday GH: “Clinical diagnosis of caliber-persistent labial artery of the lower lip. Oral Surg med oral pathol 1993 oct. $76: 4$

10. Regezi, Joseph A. Sciubba, James J. Patología bucal. 3era edición. 2.000. Interamericana MacGraw - Hill, pp 221-23 
11. Ishikawa S.: Color atlas of oral pathology, Euroamerica, inc., st. Tokio

12. Robbins: Patología estructural y funcional, Edit. Interamericana, 1974

13. Bhascar, S.N.: Patología bucal, Edit. Médica Panamericana, 1983

14. K.H. Thoma, Patología bucal, tomo II, 2da. Edición, Editorial Panamericana,1959

15. Seijas A. Ghanem A, Cedeño JC volumen 36 N³/1998 Acta Odontológica Venezolana

16. Piña V, Santos J, Sosa L., Hospital Victorino Santaella Ruiz. Mucocele - Reporte de un caso clínico en un paciente joven en la ciudad de Los Teques, Estado Miranda, Venezuela,1999.

Traducción: Fabián Fraiz

Recibido: 04-11-2011

Aceptado: 08-01-2012

Correspondencia: wilbert_chirinos@hotmail.com 